\title{
PENSAMIENTO Y ACCIÓN EN LA ENSEÑANZA DE LAS CIENCIAS
}

\author{
BAENA CUADRADO, Ma. DOLORES \\ Departamento de Didáctica e Investigación Educativa y del Comportamiento. \\ Centro Superior de Educación. Universidad de La Laguna. Tenerife.
}

\section{SUMMARY}

The educational research has studied the relationships between the teachers' thinking and their practice in Science Education. We show how to study the implicit theories and academic tasks for understanding the relationships between theory and practice in education.

\section{INTRODUCCIÓN}

Las creencias, concepciones, supuestos o teorías, que el profesorado mantiene a la hora de enfrentarse a su práctica educativa van a ser determinantes para que un modelo de enseñanza se haga realidad.

Si las concepciones que el profesorado sostiene acerca de la disciplina que enseña, cómo se produce el aprendizaje en el alumnado y cómo se diseña y desarrolla la enseñanza no son coherentes con el modelo didáctico adoptado para llevar a cabo su intervención en el aula, los resultados nunca serán los esperados.

Admitimos la existencia de una cierta coherencia entre las teorías y la acción, en cuanto que aquéllas sirven de guías orientadoras de la práctica educativa, y defendemos la necesidad de interpretar y comprender dicha práctica a partir de las concepciones teóricas de los docentes. Como afirma Carr: «Toda práctica está "incrustada en la teoría"; sólo puede comprenderse en relación con las preconcepciones teóricas "tácitas" de los practicantes» (Carr, 1990, p. 39).

En el ámbito de la didáctica de las ciencias, el tema de investigación que más ha proliferado desde los años ochenta es el de «las ideas del alumnado, sus preconcepciones y el cambio conceptual»; han ocupado, en los artículos editados en la revista Enseñanza de las Ciencias, más de un tercio de las investigaciones (Moreira, 1994). Estos estudios, además de consolidar una línea de investigación que favorece los planteamientos constructivistas provocando nuevos modelos de enseñanzaaprendizaje de las ciencias, han dirigido su atención hacia las preconcepciones docentes y epistemológicas del profesorado, desde finales de los años ochenta (Gil, 1994).

Sin embargo, el estudio del pensamiento del profesor constituye una línea de investigación que viene consolidándose desde finales de los años setenta (Clark y 
Yinger, 1979; Shavelson y Stern, 1983) y que, aunque podríamos decir que adolece de un marco global de interpretación, nos proporciona sólidas bases para intentar comprender los dilemas y los planteamientos con los que el profesorado se enfrenta en su práctica educativa.

Abordaremos, en este artículo, cómo se ha desarrollado el modelo del «pensamiento del profesor» en la investigación educativa y las limitaciones que todavía presenta este campo de estudio a la hora de relacionar la teoría con la práctica docente. Para ello, comenzaremos citando algunas investigaciones que han tratado de relacionar las concepciones e ideas del profesorado con su práctica educativa en el área de ciencias. Teniendo en cuenta las limitaciones que presentan estos estudios, propondremos el estudio de las «teorías implícitas» del profesorado, como constructo teórico que media entre el pensamiento y la acción del docente, y el de las «tareas académicas», como análisis de la organización y el contexto del aula. Estos dos ámbitos de estudio, «teorías» y «tareas», nos ofrecen respuestas a algunos de los dilemas que se plantean a la hora de comprender la relación entre el pensamiento y la acción en la enseñanza.

\section{ESTUDIOS SOBRE EL PENSAMIENTO DEL PROFESORADO EN EL AREA DE CIENCIAS Y SU RELACIÓN CON LA PRACTICA DE SU ENSENANZA}

Las investigaciones que han intentado relacionar las concepciones del profesorado de ciencias y su práctica han seguido líneas teóricas dispersas y, difícilmente, podemos llegar a conclusiones generales que nos ayuden a identificar en que sentido interacciona el pensamiento y el desarrollo de la enseñanza de los docentes.

Las concepciones mantenidas sobre el conocimiento científico y la enseñanza de las ciencias han sido analizadas desde una perspectiva que se interesa por la implicación que éstas suponen para la formación inicial y permanente del profesorado de secundaria en esta área (Hewson y Hewson, 1989), aunque estas investigaciones no incluyen observaciones del desarrollo de la enseñanza ni analizan el modelo de conocimiento pedagógico del profesorado.

El pensamiento del profesorado sobre la ciencia y las implicaciones de sus acciones en la enseñanza ha sido estudiado a partir de técnicas de observación, entrevistas y análisis de materiales por otros autores. Brickhouse (1990) defiende que una mejor y más completa formación científica del profesorado contribuye a una mejor enseñanza, aunque apunta que habría que estudiar la influencia del conocimiento sobre el contenido pedagógico en la práctica y ver cómo éste, a través de las actividades que se realizan en el aula, afecta al conocimiento científico que aprende el alumnado.

En este sentido, Gil (1993) insiste en resaltar cómo la carencia de una formación científica adecuada afecta directamente a la dificultad que presenta el profesorado a la hora de diseñar actividades innovadoras y desarrollar un currículo adecuado de ciencias, destacando la importancia del diseño de tareas como estructura de trabajo en el aula.

También se ha estudiado cómo la influencia del conocimiento pedagógico del profesorado y su relación con las concepciones epistemológicas (Porlán, 1989, 1995), a la hora de desarrollar su práctica docente, afecta directamente al conocimiento profesional que va construyendo a lo largo de su labor educativa (Porlán et al., 1996).

Algunos estudios que sí han analizado la práctica de enseñanza del profesorado de ciencias a través de tareas académicas (Mergendoller et al., 1988; Doyle, 1983; Doyle y Carter, 1984; Doyle, Sanford, Clements, French y Enmer, 1983) hacen hincapié en las demandas cognitivas que se exige al alumnado y en las implicaciones curriculares del tipo de tareas académicas, pero no aluden a la influencia que el pensamiento del profesorado tiene en la manera de estructurar dichas tareas en el aula.

La importancia de las concepciones de los docentes y los estudiantes en el análisis de tareas académicas es recogida en otras investigaciones sobre «enseñanza de las ciencias».

Sanford (1985) propone un modelo de tareas en el que incluye análisis de observaciones, de entrevistas a profesorado y alumnado, y de los materiales y documentos utilizados en el aula. Llega a la conclusión de que, para entender cuestiones importantes de la enseñanza de las ciencias, se hace necesario un estudio contextual que contenga las múltiples dimensiones que se incluyen en el sistema de trabajo en el aula y que analice, a través de las tareas, las interrelaciones de las acciones y las percepciones de profesorado y estudiantes. Su modelo se concreta en un estudio de tareas académicas sobre una unidad de genética (French y Sanford, 1985). El modelo de análisis de tareas que se utiliza resulta de gran interés, por su densidad y complejidad.

De las conclusiones que se derivan de las investigaciones que intentan profundizar en las interacciones que se producen entre el pensamiento del profesorado y la práctica de la enseñanza, podemos destacar la necesidad de considerar:

a) el «modelo pedagógico del profesorado»;

b) la utilización de las observaciones en el aula como instrumento de análisis de las tareas escolares.

Por tanto, parece de vital importancia estudiar las concepciones teóricas del profesorado a través de un constructo que aglutine tanto su «saber teórico» como «experiencial», por un lado, y el escenario concreto donde se desarrolla la acción, por otro. Estos dos aspectos (pensamiento y acción), los analizaremos a partir de las teorías implícitas del profesorado y del enfoque de tareas académicas como reflejo de la estructura y la organización del trabajo en el aula. 


\section{LAS TEORÍAS IMPLÍCITAS DEL PROFE- SORADO}

En la última década, la investigación educativa ha demostrado que el pensamiento del profesorado desempeña un papel importante en la enseñanza, proponiendo la imagen del docente como un «profesional reflexivo» que tiene sistemas de teorías y creencias susceptibles de influir en sus percepciones, planes y acciones (Zeichner, 1993; Liston y Zeichner, 1993).

Diversos estudios, desde diferentes perspectivas han intentado analizar e interpretar los procesos de pensamiento de los docentes y su relación con la práctica de la enseñanza. Clark y Peterson (1986) destacaron tres categorías principales de análisis en la investigación de dichos procesos: la planificación del docente, sus pensamientos y decisiones interactivos, y sus teorías y creencias.

En cualquier caso, la diversidad de estudios que se han elaborado en torno a un mismo paradigma, «el pensamiento del profesor», hace que sean muchos los autores que reclaman unificarlos de alguna manera, bien desde enfoques multimetodológicos que puedan captar la complejidad y multitud de facetas de la enseñanza y el aprendizaje (Kagan, 1990), bien propiciando investigaciones más genéricas que combinen procedimientos narrativos con los biográficos (Clandinin y Connelly, 1987) o, simplemente, recuperando un «sentido de comunidad» entre profesores e investigadores que trabajan juntos, como señala Elbaz (1991). El hecho es que en muy pocos casos se podría hablar de un cuerpo sistemático y acumulativo de investigación (Clark y Peterson, 1986).

Abordar el pensamiento del profesorado desde el estudio de sus «teorías implícitas» nos parece un intento acertado de unificación conceptual y metodológica, que nos ayuda a plantear aspectos relevantes en referencia a: a) la relación individuo-cultura; $b$ ) la naturaleza funcional del conocimiento, y $c$ ) las relaciones entre pensamiento y acción (Marrero, 1992).

El concepto de teoría implícita es uno de los términos usados en los estudios realizados desde la perspectiva del «pensamiento del profesor». Estas teorías vendrán prefiguradas por el conocimiento profesional del profesor, su formación didáctica e instrumental; y por el conocimiento social, cultural y ambiental en el que se desarrolla su vida escolar y personal.

El interés por el estudio de las teoría implícitas -y no de otros marcos teóricos referidos a la naturaleza del conocimiento del profesorado como «metáforas», «creencias», «perspectivas personales», «conocimiento profesional», etc.- se debe a que consideramos que el término teoría implícita hace referencia a un constructo que tiene una fundamentación cognitiva más sólida (Marrero, 1988), ya que con aquéllas no aludimos sólo a un tipo de conocimiento experiencial de carácter subjetivo, personal y situacional, sino que con estas teorías se pretende explicar la conexión entre conocimientos subjetivos y teorías formales presentes en la cultura a la cuál pertenece el profesorado.

Las teorías implícitas se consideran representaciones individuales basadas en experiencias sociales y culturales (Rodrigo, Rodríguez y Marrero, 1993; Rodrigo, 1985), siendo, pues, vínculo entre lo individual y lo social, entre lo personal y lo cultural.

En el estudio del pensamiento del profesor, nos es de especial interés considerar este vínculo, puesto que el conocimiento del profesorado es un componente mediador en la interpretación del currículo (Gimeno, 1988) y éste es considerado esencialmente un proyecto cultural, una representación de la cultura (Kemmis, 1988).

Así mismo, consideramos como socioconstructivista el proceso por el cuál se elaboran estas teorías, ya que «las teorías implícitas no se transmiten, sino que se construyen personalmente en el seno de grupos» (Rodrigo, Rodríguez y Marrero, 1993, p. 53); por tanto, se trata de una construcción personal directamente relacionada con el contexto en el que se produce. En el caso concreto del profesorado «las teorías implícitas son teorías pedagógicas personales construidas sobre la base de conocimientos pedagógicos históricamente elaborados y transmitidos a través de la formación y en la práctica pedagógica» (Marrero, 1993, p. 245).

La formación recibida por el profesorado y el contexto curricular de su práctica se integran, de forma constructiva, en sus teorías implícitas. Estas permiten a profesores y profesoras interpretar la realidad educativa y constituyen un marco de valores de referencia y de programas de acción (Marrero, 1991, 1993). Estos marcos interpretativos son flexibles y se construyen en función de las demandas de la tarea, pudiendido así hacerles frente y actuar en consecuencia. Por tanto, podemos inferir que existe un vínculo entre el pensamiento y la acción, entre la reflexión y la práctica de su enseñanza.

El estudio de las teorías implícitas, como principales intérpretes del currículo, de las innovaciones y de los intercambios académicos, constituye un conocimiento de vital importancia para entender la enseñanza y transformarla. Las concepciones implícitas que contienen los procesos de innovación pueden estar o no de acuerdo con las concepciones implícitas del profesorado, por lo que es necesario un marco de discusión y reflexión del que formen parte las diversas teorías que mantiene el profesorado de un centro. Sólo a partir de este conocimiento será posible crear un medio adecuado para el desarrollo del currículo (Marrero, 1993).

Por tanto, y de acuerdo con lo expuesto, si consideramos objetivo prioritario en educación comprender qué aspectos afectan al desarrollo de la enseñanza y cómo podemos transformar las prácticas educativas, deberemos tener en cuenta cuáles son las teorías que el profesorado mantiene y qué características presentan.

Marrero (1988) describe, en su investigación sobre «teorías implícitas y planificación del profesor», cinco tipos 
de teorías sobre la enseñanza, con las que el profesorado puede identificarse.

\section{Teoría dependiente}

- La enseñanza está guiada y dirigida por el profesorado, de tal modo que se mantiene un mismo ritmo de aprendizaje para todo el alumnado.

- Si el profesor o profesora no enseña, el alumnado por sí mismo no es capaz de aprender.

- Actitud distante con el alumnado.

- La escuela permanece aislada de los conflictos sociales y políticos.

- La enseñanza se hace dependiente de unos contenidos, del profesorado y de unos valores impuestos.

\section{Teoría productiva}

- La enseñanza es búsqueda de resultados y potenciación de la eficacia en la enseñanza y el aprendizaje.

- La enseñanza se orienta por objetivos.

- La evaluación cumple el papel de mecanismo selectivo y de control.

\section{Teoría expresiva}

- El principio de actividad es esencial.

- Importancia de: experimentación permanente, educación para la vida, cantidad de actividades, ocupación permanente del alumnado en clase.

- No existe una intención interpretativa sino un énfasis en la dimensión externalista del proceso de enseñar y aprender.

\section{Teoría interpretativa}

- Pedagogía centrada en el alumno: sus necesidades, recursos y procesos de aprendizaje.

- Actitud de búsqueda de interpretaciones de las prácticas docentes.

- Importancia de los procesos frente a los resultados.

- Destaca los aspectos comunicativos de la docencia.

\section{Teoría emancipatoria}

- Carácter moral y político.

- Índole crítica e intencionalidad emancipatoria de búsqueda de alternativas moralmente coherentes: legitima- ción contextual de ciertos objetivos y contenidos en la enseñanza, vinculación entre las prácticas de enseñanza y el marco político-social de las actuaciones de profesorado y alumnado...

- Comparte facetas metodológicas con la teoría interpretativa.

Entendemos que las teorías, en cuanto sirven de marcos de referencia para actuar, son un importante mediador en la configuración de las tareas académicas que definen la práctica en el aula; por tanto, es a la luz del estudio de las teorías implícitas como podremos entender por qué cada profesor o profesora estructura su enseñanza de una manera determinada y qué razones les lleva a hacerlo así (Baena, 1992, 1993).

Sin embargo, hasta ahora, no contábamos con estudios al respecto que nos informaran con claridad sobre qué mecanismos actúan en la transformación de una teoría, qué aspectos de la práctica docente pueden condicionar la estabilidad o la evolución de la teoría implícita que el profesorado mantiene.

Es por lo que entendíamos que se hacía necesaria una investigación a fondo del análisis de las tareas académicas que cubren el tiempo de clase, teniendo en cuenta las percepciones del alumnado y de los profesores y profesoras, y partiendo de las teorías implícitas que definen el pensamiento del docente (Baena, 1995).

\section{LAS TAREAS ACADÉMICAS. MODELO DE ANÁLISIS DEL AULA}

Toda práctica de enseñanza y sus efectos educativos se valoran en función del escenario concreto donde se desarrollan. La investigación sobre educación debe comprender qué significados aporta el estudio de las prácticas reales en la enseñanza. Las tareas, que se realizan en el aula no siempre han sido estudiadas como parte fundamental de la práctica; sólo un modelo que tenga en cuenta cómo se llevan a cabo tareas concretas en el aula y cómo éstas están relacionadas, por una parte, con las decisiones que el profesorado toma acerca de la acción pedagógica $y$, por otro, con lo que los estudiantes pueden aprender constituirá un marco de investigación que permita comprender la enseñanza y buscar vías para transformarla y mejorarla.

Tres aspectos definirían el concepto de tarea (Doyle, 1983; Doyle y Carter, 1984): un producto o meta, una serie de recursos disponibles en cada situación y una serie de operaciones que se aplican sobre los recursos para conseguir dicha meta.

Partiendo del supuesto de que el análisis detallado de lo que ocurre en el aula nos dará pautas para interpretar los comportamientos de profesorado y alumnado, sus actitudes, los efectos en términos de aprendizaje... se hace necesario encontrar un modelo útil de análisis de la vida de aula. 
Doyle aporta el «modelo de tareas» dentro de lo que podríamos llamar el paradigma ecológico en investigación educativa (Doyle, 1977, 1979; Bromfenbrenner, 1979; Pérez Gómez, 1983, 1992).

Este enfoque de investigación exige, en líneas generales, una perspectiva metodológica que suponga, al menos: un énfasis en las observaciones a largo plazo, un interés en la descripción de los procesos que se desarrollan en el aula y una preocupación por la perspectiva de los participantes acerca de los hechos (Doyle, 1981).

De esta manera será posible identificar los dos subsistemas que condicionan la vida en el aula: la estructura de tareas académicas y la estructura social de participación (Doyle, 1977). Todos los acontecimientos que se producen en estas dos estructuras adquieren un carácter intencional y evaluador.

Desde la perspectiva del alumnado, la estructura de tareas académicas está contextualizada en un sistema de evaluación, bajo condiciones de ambigüedad y riesgo (Doyle, 1979, 1983, 1985); ambigüedad referida a si se conoce o no el tipo de respuesta que se espera, y riesgo con referencia a las consecuencias que tendrá su respuesta.

Gimeno (1988) encuentra en las tareas diferentes funciones que van desde ser mediadoras de la calidad de la enseñanza hasta ser útiles en la formación y perfeccionamiento del profesorado, pasando por considerarlas como base de análisis de la profesionalidad docente y de comunicación entre teoría, conocimiento subjetivo y práctica. Estas características definitorias de las tareas junto con las ya presentadas en el modelo de Doyle hacen elevar el concepto de tarea académica hasta el nivel de «escenario dinámico de interacción pedagógica» (Baena, 1995).

Las tareas conforman el escenario donde interactúan los procesos curriculares. No limitándose su influencia a la práctica inmediata sino que afectan también a la complejidad de la acción docente y a la absorción, por parte de profesorado y alumnado, de los valores explícitos e implícitos que se generan.

Las tareas constituyen una adecuada unidad de análisis de la práctica en la medida que «una cierta secuencia de unas cuantas de ellas constituyen un modelo metodológico, acotando el significado real de un proyecto de educación que pretende unas metas y que se guía por ciertas finalidades» (Gimeno, 1988, p. 248).

Con respecto a la profesionalidad del docente, la elección entre un tipo u otro de tareas vendrá determinado, según Gimeno (1988), por los dilemas prácticos que se plantean al profesorado en función de la interacción entre sus condicionamientos individuales y los contextuales. La estructura de tareas académicas será un reflejo fiel de hasta qué punto están influyendo unos u otros condicionamientos en el desarrollo de su profesión.

Nosotros, además, defendemos que estos dilemas prácticos vienen determinados, en gran medida, por las teorías implícitas que el profesorado mantiene. De esta forma, estudiando la manera en que se desarrollan las tareas académicas en el aula, podremos aventurar hasta qué punto influyen las teorías implícitas del profesor o profesora en la toma de decisiones.

Un análisis de tareas nos mostrará la coherencia teóricopráctica de la enseñanza, ya que éstas hacen de vehículo entre los supuestos teóricos y la acción. Además lo hacen en los dos sentidos, cómo esquemas teóricos de acción a poner en práctica y como reflexión teórica a partir de situaciones prácticas.

El análisis de las tareas que se desarrollan en la vida de un aula estudiado desde esta perspectiva, junto con las aportaciones personales que tanto profesorado como alumnado pueden ofrecer, nos ayuda a mostrar no sólo lo que ocurre en una realidad concreta sino también a encontrar pautas generales sobre las razones de por qué ocurre y los puntos claves donde el cambio se hace necesario.

\section{ANÁLISIS DE TEORÍAS IMPLÍCITAS Y TAREAS ACADEMICAS EN UN ESTUDIO DE CASOS}

Bajo los supuestos teóricos desarrollados en los apartados anteriores, llevamos a cabo una investigación en la que se analiza en profundidad el modelo de tareas académicas de dos profesoras del área de ciencias en educación secundaria (Baena, 1995).

Nuestro interés se centra en analizar la práctica de enseñanza del profesorado, así como las características que definen sus teorías implícitas, con el fin de establecer relaciones entre pensamiento y acción fundamentalmente.

Para poder describir y comprender en profundidad contextos de enseñanza concretos, escogemos el estudio de casos como modelo de investigación dentro de la perpectiva interpretativa (Hamilton, 1983; Walker, 1983; Goetz y LeCompte, 1988; Marcelo et al., 1991).

Estas profesoras fueron seleccionadas para el estudio por su identificación con rasgos de teorías implícitas muy diferenciadas entre sí, es decir, una de ellas (Julia) representaba el pensamiento caracterizado por teorías dependiente y productiva, principalmente; la otra (Andrea), por su similitud con las teorías interpretativa y emancipatoria.

Para estudiar y analizar la forma en que estas profesoras estructuran las tareas académicas y saber qué estructura social de participación implica dicha organización, se realizaron observaciones en el aula durante un curso académico completo. Así mismo, se recogió información a través de otros procedimientos como: entrevistas a cada una de las profesoras durante todo el año, entrevistas al alumnado y análisis de los materiales que se utilizaron en dicho curso. 
El análisis de los aspectos fundamentales mencionados (pensamiento, acción y aprendizaje) nos ayuda a desvelar los interrogantes que nos preocupaban al iniciar la investigación:

a) ¿Qué relación existe entre el pensamiento y la acción (ideas y tareas)?

b) ¿Cómo se organizan las tareas (acción)?

c) ¿Qué relación existe entre las tareas y el aprendizaje?

El modelo general de análisis de la investigación (como se representa en la figura 1) nos conduce a la elaboración de tres procesos de análisis (pensamiento-acción, acción y acción-aprendizaje) en los que interaccionan los resultados obtenidos:

1) Proceso de análisis de la acción. Incluye, por un lado, el análisis de las actividades desarrolladas desde un punto de vista organizativo y de contexto; $y$, por otro lado, el análisis de las tareas, su nivel de estructuración y significado.

2) Proceso de análisis del pensamiento-acción. Se analizan las tareas principales (que incluyen las actividades y tareas de cada trimestre del curso), las entrevistas realizadas a las profesoras y el cuestionario de teorías implícitas.

3) Proceso de análisis de la acción-aprendizaje. Se lleva a cabo un análisis global de todo el curso, se analizan las entrevistas mantenidas con el alumnado y los documentos y materiales escritos proporcionados, tanto por las profesoras como los utilizados en el aula.

Como se puede observar, el proceso de análisis de la acción es el eje fundamental que interacciona con los demás procesos de análisis. Como nuestro estudio se extiende durante todo un año académico, nos vimos ante la necesidad de definir diferentes niveles de análisis de la acción, que se integran progresivamente hasta conseguir el análisis global del curso completo.

El primer nivel de análisis de la acción es el de «actividad» definida como una unidad mínima de acción organizada, con un objetivo a conseguir y delimitada en el tiempo. Las dimensiones estudiadas a este nivel se refieren a la organización y al contexto de dicha actividad.

El segundo nivel de análisis sería el de «tarea» definida como una secuencia de actividades (pertenecientes a una

Figura 1

Modelo general de análisis de la investigación.

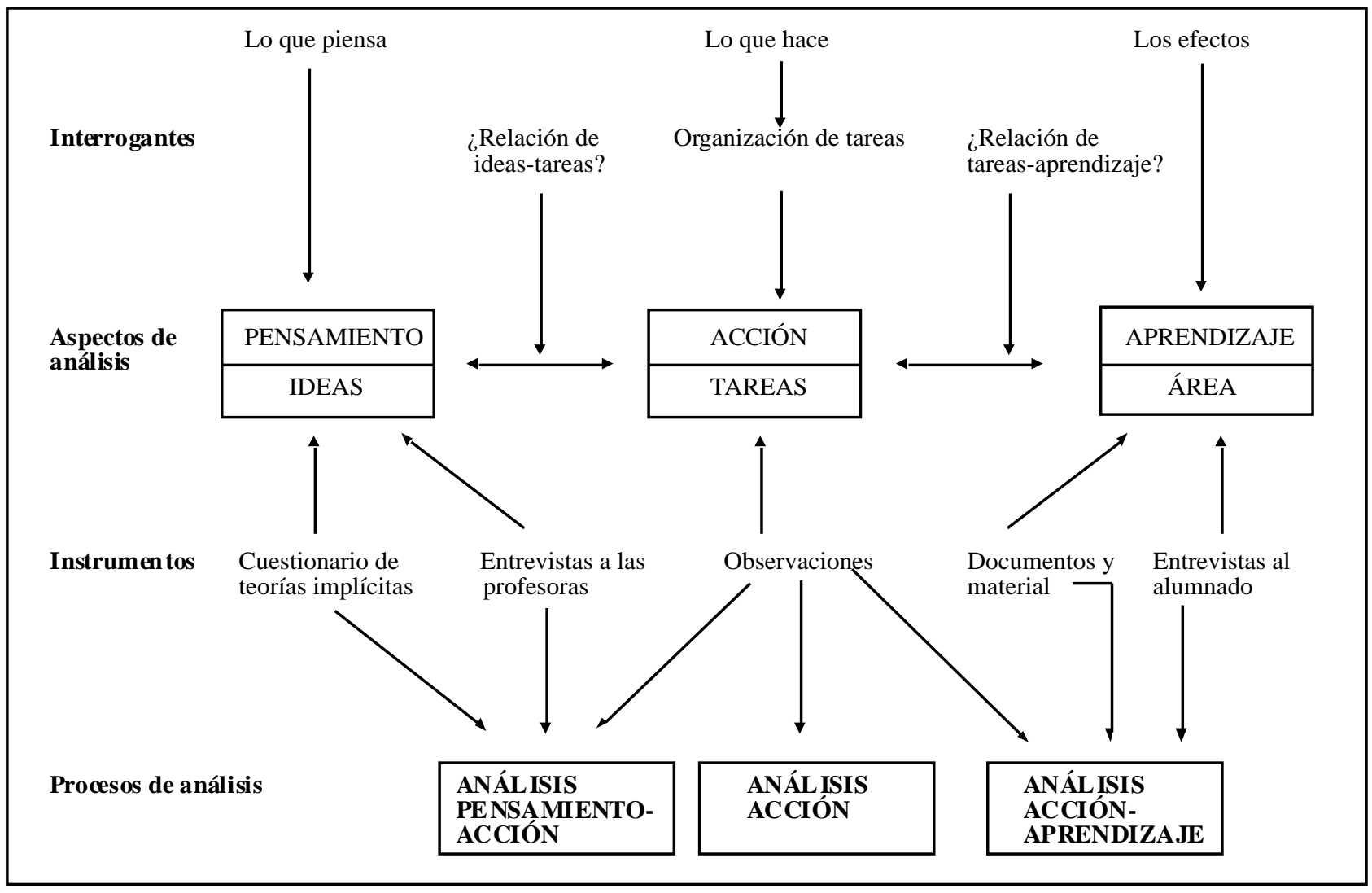


misma o a distintas sesiones de clase) en la que se ha trabajado el mismo contenido temático. Aquí daremos cuenta de las dimensiones referidas tanto a la estructuración de la tarea (contenido temático, temporalización y actividades que la constituyen) como al significado de la misma (contenido, tiempo, función docente y clima del aula).

El tercer nivel de análisis es el de la «tarea principal» entendida como la agrupación de tareas que constituyen una secuencia lógica o coherente de contenidos, es decir, que reflejan un eje teórico central de referencia o, en su defecto, que están agrupadas en espacios de tiempo prolongados (trimestre).

Estos tres niveles de análisis incluyentes culminan en el análisis global del curso. En este nivel se presenta una relación de todas las tareas y las actividades desarrolladas en los tres trimestres del curso, se analiza y discuten las dimensiones señaladas en todas las actividades desarrolladas. Con estos datos diseñamos el patrón de desarrollo instructivo que siguen las profesoras y el esquema de estructuración que se lleva a cabo en los tres trimestres del curso, lo que nos lleva al modelo metodológico que presenta cada profesora.

Tanto el nivel de análisis de la «tarea principal» como el del curso se ve complementado con los datos obtenidos en las estrevistas a profesoras y alumnado, con el análisis de los materiales utilizados en clase y con los resultados obtenidos en el «cuestionario de teorías implícitas», para finalmente completar el modelo de enseñanza que cada una de las profesoras configura con su propia práctica, modelo que presenta unas concepciones de la enseñanza desde el punto de vista epistemológico, psicológico y didáctico.

A continuación, presentamos los resultados finales a los que el análisis de la práctica nos ha conducido: la estructuración seguida por las profesoras, sus modelos metodológicos y las concepciones sobre la enseñanza de las ciencias, regidas por principios epistemológicos, psicológicos y didácticos, que definen su propio modelo de enseñanza.

\section{EL MODELO DE ENSEÑANZA DE JULIA}

a) Estructuración de la práctica en cada trimestre

\begin{tabular}{|l|l|l|}
\hline \multicolumn{2}{|c|}{ ESTRUCTURACIÓN } \\
\hline TRIMESTRE 1 ${ }^{\circ}$ & \multicolumn{1}{|c|}{ TRIMESTRE 2 } & TRIMESTRE 3 \\
$\begin{array}{l}\text { - Emisión de } \\
\text { información por } \\
\text { medio de explica- } \\
\text { ciones o lectura } \\
\text { del libro por parte } \\
\text { de la profesora, } \\
\text { lectura del } \\
\text { alumnado o } \\
\text { proyección de }\end{array}$ & $\begin{array}{l}\text { información de } \\
\text { de la profesora } \\
\text { o de la lectura del } \\
\text { libro de texto por } \\
\text { parte del } \\
\text { alumnado }\end{array}$ & $\begin{array}{l}\text { por parte de la } \\
\text { profesora }\end{array}$ \\
\hline
\end{tabular}

\begin{tabular}{|c|c|c|}
\hline $\begin{array}{l}\text { vídeo (en una } \\
\text { ocasión) }\end{array}$ & & \\
\hline $\begin{array}{l}\text { - Preguntas y } \\
\text { respuestas } \\
\text { por escrito }\end{array}$ & $\begin{array}{l}\text { - Preguntas y } \\
\text { respuestas } \\
\text { por escrito }\end{array}$ & \\
\hline $\begin{array}{l}\text { - Corrección de } \\
\text { ejercicios }\end{array}$ & $\begin{array}{l}\text { - Corrección } \\
\text { de ejercicios }\end{array}$ & $\begin{array}{l}\text { - Corrección de } \\
\text { ejercicios }\end{array}$ \\
\hline $\begin{array}{l}\text { - Prueba de } \\
\text { control }\end{array}$ & $\begin{array}{l}\text { - Prueba de } \\
\text { control }\end{array}$ & - Prueba de control \\
\hline
\end{tabular}

b) Modelo metodológico global del curso

\begin{tabular}{|l|l|}
\hline $\begin{array}{c}\text { Secuenciación de fases } \\
\text { metodológicas }\end{array}$ & Actividades realizadas \\
\hline $\begin{array}{l}\text { Información-presentación } \\
\text { del material }\end{array}$ & $\begin{array}{l}\text { Exposición de la profesora. } \\
\text { Lectura del libro de texto } \\
\text { por parte de la profesora } \\
\text { o el alumnado } \\
\text { Proyección de vídeo }\end{array}$ \\
\hline $\begin{array}{l}\text { Actividades del alumnado } \\
\text { para resolver conflictos }\end{array}$ & Ejercicios escritos \\
\hline $\begin{array}{l}\text { Consolidación de los } \\
\text { conocimientos }\end{array}$ & $\begin{array}{l}\text { Corrección de ejercicios } \\
\text { Pruebas de control }\end{array}$ \\
\hline
\end{tabular}

c) Síntesis de los principios epistemológicos, psicológicos y didácticos del modelo de enseñanza de la profesora Julia.

\begin{tabular}{|c|c|}
\hline \multicolumn{2}{|c|}{$\begin{array}{c}\text { CONCEPCIONES DE LA ENSEÑANZA } \\
\text { DE LAS CIENCIAS }\end{array}$} \\
\hline $\begin{array}{l}\text { EPISTEMO- } \\
\text { LÓGICAMENTE }\end{array}$ & $\begin{array}{l}\text { Ciencia como disciplina neutral y objetiva } \\
\text { Actividad independiente de las condicio- } \\
\text { nes históricas y sociales } \\
\text { Conocimiento científico inmutable }\end{array}$ \\
\hline $\begin{array}{l}\text { PSICOLÓGI- } \\
\text { CAMENTE }\end{array}$ & $\begin{array}{l}\text { Aprendizaje lineal y acumulativo } \\
\text { No se establece conexión con las ideas del } \\
\text { alumnado } \\
\text { Desconexión con la realidad. } \\
\text { No se aprende en diferentes contextos }\end{array}$ \\
\hline $\begin{array}{l}\text { DIDÁCTICA- } \\
\text { MENTE }\end{array}$ & $\begin{array}{l}\text { Objetivos que potencian la individualidad } \\
\text { y la acumulación de contenidos } \\
\text { conceptuales } \\
\text { Selección de contenidos guiados por el } \\
\text { libro de texto } \\
\text { Secuenciación y organización de } \\
\text { contenidos lineales. No se integran ni } \\
\text { relacionan unos contenidos con otros } \\
\text { Estructuración metodológica que } \\
\text { favorece el individualismo y la memori- } \\
\text { zación de conceptos, considerando los } \\
\text { procedimientos y actitudes irrelevantes } \\
\text { Criterios de evaluación cerrados. } \\
\text { Relevancia del orden y la disciplina }\end{array}$ \\
\hline
\end{tabular}




\section{EL MODELO DE ENSEÑANZA DE ANDREA}

a) Estructuración de la práctica en cada trimestre

\begin{tabular}{|c|c|c|}
\hline \multicolumn{3}{|c|}{ ESTRUCTURACIÓN } \\
\hline TRIMESTRE $1^{\circ}$ & TRIMESTRE $2^{\circ}$ & TRIMESTRE $3^{\circ}$ \\
\hline $\begin{array}{l}\text { - Elaboración } \\
\text { individual, por } \\
\text { parte del alumnado, } \\
\text { de un cuestionario } \\
\text { sobre los cuatro } \\
\text { temas señalados } \\
\text { del libro de texto } \\
\text { - Formación de } \\
\text { pequeños grupos } \\
\text { de trabajo } \\
\text { (3 ó } 4 \text { componentes) } \\
\text { donde se pone } \\
\text { en común el } \\
\text { cuestionario } \\
\text { individual } \\
\text { - Elaboración de } \\
\text { un cuestionario } \\
\text { común para toda } \\
\text { la clase, elaborado } \\
\text { por la profesora } \\
\text { a partir del material } \\
\text { presentado por } \\
\text { el alumnado }\end{array}$ & $\begin{array}{l}\text { - Planteamiento } \\
\text { de problemas } \\
\text { por parte de la } \\
\text { profesora. } \\
\text { Discusión }\end{array}$ & $\begin{array}{l}\text { - Elaboración de un } \\
\text { cuestionario común } \\
\text { para toda la clase, } \\
\text { por parte de la } \\
\text { profesora a partir } \\
\text { del material } \\
\text { presentado por el } \\
\text { alumnado }\end{array}$ \\
\hline $\begin{array}{l}\text { - Investigación } \\
\text { individual y por } \\
\text { grupos sobre los } \\
\text { contenidos: } \\
\text { búsqueda de } \\
\text { bibliografía, } \\
\text { realización de } \\
\text { experimentos, } \\
\text { discusiones }\end{array}$ & $\begin{array}{l}\text { - Trabajo indivi- } \\
\text { dual, en peque- } \\
\text { ño grupo y en } \\
\text { gran grupo sobre } \\
\text { los problemas } \\
\text { propuestos. Dis- } \\
\text { cusión y debate }\end{array}$ & $\begin{array}{l}\text { - Trabajo individual } \\
\text { y por grupos, para } \\
\text { dar respuesta a los } \\
\text { interrogantes } \\
\text { propuestos en el } \\
\text { cuestionario }\end{array}$ \\
\hline $\begin{array}{l}\text { - Ejercicios en } \\
\text { clase y en casa, } \\
\text { propuestos por la } \\
\text { profesora }\end{array}$ & $\begin{array}{l}\text { - Información } \\
\text { verbal y escrita } \\
\text { sobre el tema, por } \\
\text { parte de la profe- } \\
\text { sora }\end{array}$ & $\begin{array}{l}\text { - Exposición por } \\
\text { parte de la profesora } \\
\text { y el alumnado de } \\
\text { algunos contenidos } \\
\text { concretos que } \\
\text { consideran de } \\
\text { interés }\end{array}$ \\
\hline $\begin{array}{l}\text { - Prueba de control } \\
\text { escrita, con material } \\
\text { de consulta }\end{array}$ & $\begin{array}{l}\text { - Resolución de } \\
\text { problemas. Crea- } \\
\text { ción de nuevos } \\
\text { interrogantes }\end{array}$ & $\begin{array}{l}\text { - Realización de } \\
\text { actividades de } \\
\text { investigación para } \\
\text { elaborar un informe } \\
\text { interdisciplinar } \\
\text { sobre un ecosistema }\end{array}$ \\
\hline
\end{tabular}

b) Modelo metodológico global durante el curso

\begin{tabular}{|l|l|}
\hline \multicolumn{1}{|c|}{$\begin{array}{c}\text { Secuenciación de fases } \\
\text { metodológicas }\end{array}$} & Actividades realizadas \\
\hline Información-presentación & Información general \\
del material & sobre contenidos a estudiar \\
& Búsqueda bibliográfica \\
& Elaboración de cuestionarios \\
& Planteamiento de problemas \\
& Exposición profesora-alumnado \\
\hline
\end{tabular}

\begin{tabular}{|l|l|}
$\begin{array}{l}\text { Actividades del alumnado } \\
\text { para resolver conflictos }\end{array}$ & $\begin{array}{l}\text { Realización de experimentos } \\
\text { Exposición de experimentos } \\
\text { Discusión-debate } \\
\text { Ejercicios escritos }\end{array}$ \\
\hline $\begin{array}{l}\text { Consolidación de los } \\
\text { conocimientos }\end{array}$ & $\begin{array}{l}\text { Evaluación de todas las } \\
\text { actividades } \\
\text { Corrección de ejercicios } \\
\text { Pruebas de control con } \\
\text { material de consulta } \\
\text { Actividades de investigación }\end{array}$ \\
\hline
\end{tabular}

c) Síntesis de los principios epistemológicos, psicológicos y didácticos del modelo de enseñanza de la profesora Andrea.

\begin{tabular}{|l|l|}
\hline CONCEPCIONES DE LA ENSENANZA DE LAS CIENCIAS \\
\hline $\begin{array}{l}\text { EPISTEMOLÓ- } \\
\text { GICAMENTE }\end{array}$ & $\begin{array}{l}\text { Ciencia como construcción social } \\
\text { Actividad condicionada por la } \\
\text { comunidad científica y con utilidad social } \\
\text { Relatividad del conocimiento científico, } \\
\text { al que se puede acceder desde } \\
\text { diferentes perspectivas }\end{array}$ \\
\hline PSICOLÓGI- & $\begin{array}{l}\text { Aprendizaje como construcción } \\
\text { activa, teniendo en cuenta las opiniones } \\
\text { e intereses del grupo }\end{array}$ \\
$\begin{array}{l}\text { Importancia de los intereses del } \\
\text { alumnado respecto al nuevo conocimiento }\end{array}$ \\
$\begin{array}{l}\text { Elaboración del conocimiento en } \\
\text { diferentes contextos, potenciando la } \\
\text { utilidad del saber }\end{array}$ \\
\hline MENTE & $\begin{array}{l}\text { Objetivos relacionados con la integración } \\
\text { social y la aceptación de diferentes valores } \\
\text { y opiniones }\end{array}$ \\
$\begin{array}{l}\text { Selección de contenidos, teniendo en } \\
\text { cuenta la opinión del alumnado y sus } \\
\text { motivaciones }\end{array}$ \\
$\begin{array}{l}\text { Secuenciación y organización de } \\
\text { contenidos coherentes con la lógica de } \\
\text { la disciplina: relacionando diferentes } \\
\text { núcleos conceptuales e integrando unos } \\
\text { contenidos con otros }\end{array}$ \\
$\begin{array}{l}\text { Estructuración metodológica que favorece } \\
\text { la expresión personal, la discusión entre } \\
\text { iguales y el desarrollo de iniciativas. } \\
\text { Se le concede similar importancia a los } \\
\text { conceptos, procedimientos y actitudes }\end{array}$ \\
$\begin{array}{l}\text { La evaluación se adapta al proceso de } \\
\text { enseñanza-aprendizaje }\end{array}$
\end{tabular}

Vemos que, en el caso de Julia, la estructuración y metodología seguidas permanecen invariables a lo largo del curso, independientemente del contenido curricular que se trabaje o de las dificultades de aprendizaje que presente el alumnado. 
Los principios epistemológicos, psicológicos y didácticos que definen sus concepciones sobre la enseñanza de las ciencias demuestran la similitud entre el modelo de enseñanza de Julia y los modelos más tradicionales de enseñanza: «modelo como presentación de conocimientos elaborados» (Baena, 1995), lo que demuestra la pervivencia de prácticas de enseñanza que «teóricamente» parecen estar superadas, pero que en la realidad conviven con otras prácticas más innovadoras.

En el caso de Andrea, por el contrario, hemos visto cómo la distribución y estructuración de actividades y tareas a lo largo del curso varía sensiblemente. La metodología desarrollada se modifica, adaptándose a: las dificultades de aprendizaje, la familiaridad del alumnado con el trabajo, el tiempo del que se dispone o los intereses que van surgiendo en el transcurso de la acción.

El modelo de enseñanza-aprendizaje que subyace en la estructuración de tareas que hemos identificado en la práctica docente de Andrea responde a unos principios epistemológicos, psicológicos y didácticos que se asemejan bastante a los defendidos por el modelo «construcción y reconstrucción del conocimiento» en la enseñanza de las ciencias (Baena, 1995).

En definitiva, podemos llegar a la conclusión de que, cuando el pensamiento del profesorado está vinculado a teorías implícitas más tradicionales («dependiente», «productiva») caracterizadas por ser más simples y con rasgos definitorios más rígidos, su práctica denota actividades más rígidas, lineales y sin grandes modificaciones. Sin embargo, cuando las teorías implícitas que se mantienen son más innovadoras («interpretativa», «emancipatoria»), con características más flexibles y complejas, las actividades tienen una estructuración más flexible y ambigua, con una estructuración de la práctica compleja, no identificable con un patrón constante del desarrollo de la acción en el aula.

\section{REFERENCIAS BIBLIOGRÁFICAS}

BAENA, M.D. (1992). Teorías implícitas del profesor, tareas académicas y enseñanza de las ciencias, en Estebaranz, A., Sánchez, V. (eds.). Pensamiento de profesores y desarrollo profesional I, pp. 49-56. Secr. de Publicaciones de la Universidad de Sevilla.

BAENA, M.D. (1993). Interacción teoría-práctica en el profesorado de ciencias. Dos estudios de casos. Qurriculum, 6-7, pp. 121-137.

BAENA, M.D. (1995). Tareas académicas y teorías implícitas del profesorado. Estudio de casos en la enseñanza de las ciencias. Tesis doctoral. Departamento de Didáctica e Investigación Educativa y del Comportamiento. Universidad de La Laguna.

BRICKHOUSE, N.W. (1990). Teachers' beliefs about the nature of science and their relationship to classroom practice. Journal of Teacher Education, 41(3), pp. 53-62.

BRONFENBRENNER, U. (1979). The ecology of human development. Harvard University Press.

\section{CONCLUSIONES}

Como conclusión general, podríamos decir que, cuando queremos analizar las relaciones entre aspectos tan complejos como pueden ser el pensamiento del profesorado y la práctica de la enseñanza, no podemos simplificar el estudio a una simple asociación entre las creencias u opiniones del profesorado y los rasgos externos que caracterizan su acción en el aula.

La manera de pensar del docente está impregnada de toda una serie de variables personales y profesionales que van conformando su modelo teórico y práctico de la enseñanza. Analizando en profundidad las dimensiones que configuran un modo de actuar y las repercusiones que éste tiene en el aprendizaje que se genera en el alumnado, tanto desde el punto de vista académico como social, podremos concretar los puntos de conexión entre la teoría y la práctica para así poder elaborar modelos de formación y asesoramiento del profesorado que ayuden a mejorar la calidad docente.

Para que se modifiquen las prácticas de la enseñanza consideramos indispensable que el profesorado sea consciente de los rasgos que definen su pensamiento y de cómo éste está afectando a la calidad de su enseñanza. Se hace necesario una transformación de teorías para transformar la práctica. Ésta no se modificará si el profesorado no analiza su propia práctica y toma conciencia de las contradicciones de su modelo de enseñanza.

Un nuevo modelo de enseñanza nunca se adoptará por imposición sino por el convencimiento de la necesidad de cambio de la práctica docente.

CARR, W. (1990). Hacia una ciencia crítica de la educación. Barcelona: Laertes

CLANDININ, D.J.y CONELLY,F.M.(1987). Teachers' personal knowledge: what counts as personal in studies of the personal. Journal Curriculum Studies, 19(6), pp. 487-500.

CLARK, C.M. y INGER, R.J. (1979). Teachers' thinking, en Peterson, P., Walberg, H.J. (eds.). Research on Teaching. Concepts, Finding and Implications, pp. 231-263. Berkeley: McCutchan Publishing Corporations.

CLARK, C.M. y PETERSON, P.L. (1990). Procesos de pensamiento de los docentes, en Wittrock, M.C. La investigación de la enseñanza, III. Barcelona: Paidós.

DOYLE, W. (1977). Paradigms for Research on Teacher Effectiveness, en Shulman, L.S. Review of Research in Education, 5, pp. 163-198. Illinois: Peacook. Itaca.

DOYLE, W. (1979). Classroom Tasks and Students' Abilities, en Peterson, P.L., Walberg, H.J.McCatchin, Berkeley. Research 
on Teaching: Concepts, Studings and Implications, pp. 183209.

DOYLE, W. (1981). La investigación sobre el contexto del aula: hacia un conocimiento básico para la práctica y política de formación del profesorado. Revista de Educación, 277, pp. 29-42. Madrid: MEC.

DOYLE, W. (1983). Academic work. Review of Educational Research, 53(2), pp. 159-199.

DOYLE, W. y CARTER, K. (1984). Academic Tasks in Classrooms. Curriculum Inquiry, 14(2), pp. 129-149.

DOYLE, W., SANFORD, J., CLEMENTS, B., FRENCH, B. S. y ENMER, E. (1983). Managing academic tasks: an interin report of the junior high study. Austin: Research and Development Center for Teacher Education. The University of Texas at Austin.

DOYLE, W.(1985). Recent research on classroom management: implications for teacher preparation. Journal of Teacher Education, 36(3), pp. 31-35.

ELBAZ, F. (1991). Research on teachers' knowledge: the evolution of a discourse. Journal Curriculum Studies, 23(1), pp. 1-20.

FRENCH, B.A.S. y SANFORD, J.P. (1985). Academic Tasks in High School Biology: a genetics unit. Washington, DC: National Inst. of Education (ed.).

GIL, D. (1993). Aportaciones de la investigación en la didáctica de las ciencias a la formación y actividad del profesorado. Qurriculum, 6-7, pp. 45-66.

GIL, D. (1994). Diez años de investigación en didáctica de las ciencias: realizaciones y perspectivas. Enseñanza de las Ciencias, 12(2), pp. 154-164.

GIMENO, J. (1988). El currículo: una reflexión sobre la práctica. Madrid: Morata.

GOETZ, J.P. y LECOMPTE, M.D. (1988). Etnografía y diseño cualitativo en investigación educativa. Madrid: Morata.

HAMILTON, D. (1983). Contrastes de supuestos entre el análisis de muestras y el estudio de casos en la investigación, en Gimeno, J. y Pérez, A. La enseñanza: su teoría y su práctica. Madrid: Akal.

HEWSON, P.W.y HEWSON, M.G. (1989). Analysis and use of a task for identifying conceptions of teaching science. Journal of Education for Teaching, 15(3), pp. 191-209.

KAGAN, D. (1990) Ways of Evaluating Teacher Cognition: Inferences Concerning the Goldilocks Principle. Reviews of Educational Research, 60(3), pp. 419-469.

KEMMIS, S. (1988). El currículo: más allá de la teoría de la reproducción. Madrid: Morata.

LISTON, D. y ZEICHNER, K.M. (1993). La formación del profesorado y las condiciones sociales de la enseñanza. Madrid: Morata.

MARCELO, C. et al. (1991). El estudio de caso en la formación del profesorado y la investigación didáctica. Secret. de Publicaciones de la Universidad de Sevilla.
MARRERO, J. (1988). Teorías implícitas del profesor y planificación de la enseñanza. Tesis doctoral. Departamento de Didáctica e Investigación Educativa y del Comportamiento. Universidad de La Laguna.

MARRERO, J. (1992) Las teorías implícitas del profesorado: un puente entre la cultura y la práctica de la enseñanza, en Estebaranz, A., Sánchez, V. (eds.). Pensamiento de profesores y desarrollo profesional I, pp. 9-21 Secr. de Publicaciones de la Universidad de Sevilla.

MARRERO, J. (1993). Las teorías implícitas del profesorado: vínculo entre la cultura y la práctica de la enseñanza, en Rodrigo, M.J., Rodríguez, A., Marrero, J. Las teorías implícitas. Una aproximación al conocimiento cotidiano, pp. 243-276. Madrid:Visor.

MERGENDOLLER et al. (1988). Tasks demands and accountability in middle-grade sciences classes. The elementary school Journal, 88(3), pp. 251-265.

MOREIRA, M.A. (1994) Diez años de la revista Enseñanza de las ciencias: de una ilusión a una realidad. Enseñanza de las Ciencias, 12(2), pp. 147-153.

PÉREZ GÓMEZ, A.I. (1983). Paradigmas contemporáneos de investigación educativa, en Gimeno, J., Pérez, A. La enseñanza: su teoría y su práctica, pp. 95-138. Madrid: Akal.

PÉREZ GÓMEZ, A. (1992). Comprender la enseñanza en la escuela. Modelos metodológicos de investigación educativa, en Gimeno, J., Pérez, A. Comprender y transformar la enseñanza. Madrid: Morata.

PORLÁN, R. (1989). Teoría del conocimiento, teoría de la enseñanza y desarrollo profesional. Las concepciones epistemológicas de los profesores. Tesis doctoral. Universidad de Sevilla.

PORLÁN, R. (1995). Las creencias pedagógicas y científicas de los profesores. Enseñanza de las ciencias de la Tierra, 3(1), pp. 7-13.

PORLÁN, R. et al. (1996). Conocimiento profesional deseable y profesores innovadores: fundamentos y principios formativos. Investigación en la Escuela, 29, pp. 23-38.

RODRIGO, M.J.(1985). Las teorías implícitas en el conocimiento social. Infancia y Aprendizaje, 31-32, pp. 145-156.

RODRIGO, M.J., RODRÍGUEZ, A. y MARRERO, J. (1993). Las teorías implícitas. Una aproximación al conocimiento cotidiano. Madrid:Visor.

SANFORD, J.P. (1985). Academic tasks and research in Science Teaching.Texas Univ., Austin. Research and Development Center for Teacher Education.

SHAVELSON, R.J. y STERN, P. (1983). Investigación sobre el pensamiento pedagógico del profesor, sus juicios, decisiones y conductas, en Gimeno, J., Pérez, A. La enseñanza: $s u$ teoría y su práctica, pp. 372-419. Madrid: Akal.

WALKER, R. (1983). La realización de estudios de casos en educación. Ética, teoría y procedimientos, en Dockrell, W.B., Hamilton, D. Nuevas reflexiones sobre la investigación educativa, pp. 42-88. Madrid: Narcea,

ZEICHNER, K.M. (1993)El maestro como profesional reflexivo. Cuadernos de Pedagogía, 220, pp. 44-49. 